\title{
The roles of atomic and molecular gas on the redshift evolution of star formation and metallicity in galaxy formation models
}

\author{
Jian Fu and Guinevere Kauffmann \\ Max-Planck-Institut für Astrophysik, D-85741 Garching bei München, Germany \\ email: fujian@mpa-garching.mpg.de
}

\begin{abstract}
We study the redshift evolution of neutral and molecular gas in the interstellar medium with the results from semi-analytic models of galaxy formation and evolution, which track the cold gas related physical processes in radially resolved galaxy disks. Two kinds of prescriptions are adopted to describe the conversion between molecular and neutral gas in the ISM: one is related to the gas surface density and gas metallicity based on the model results by Krumholz, Mckee \& Tumlinson; the other is related the pressure of ISM. We try four types of star formation laws in the models to study the effect of the molecular gas component and the star formation time scale on the model results, and find that the $\mathrm{H}_{2}$ dependent star formation rate with constant star formation efficiency is the preferred star formation law. We run the models based on both Millennium and Millennium II Simulation haloes, and the model parameters are adjusted to fit the observations at $z=0$ from THINGS/HERACLES and ALFALFA/COLD GASS. We give predictions for the redshift evolution of cosmic star formation density, $\mathrm{H}_{2}$ to HI cosmic ratios, gas to star mass ratios and gas metallicity vs stellar mass relation. Based on the model results, we find that: (i) the difference in the $\mathrm{H}_{2}$ to $\mathrm{HI}$ ratio at $z>3$ between the two $\mathrm{H}_{2}$ fraction prescriptions can help future observations to test which prescription is better; (ii) a constant redshift independent star formation time scale will postpone the star formation processes at high redshift and cause obvious redshift evolution for the relation between gas metallicity and stellar mass in galaxies at $z<3$.
\end{abstract}

Keywords. galaxies: evolution - ISM: atoms - ISM: molecules — stars: formation
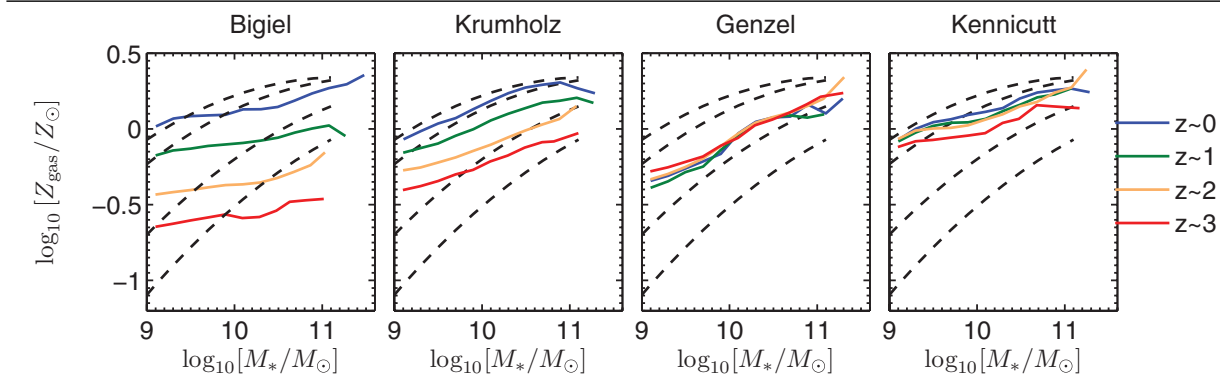

Figure 1. The relation between gas-phase metallicity and stellar mass for star-forming galaxies is plotted at different redshifts. The 4 panels show results for the 4 star formation models, which can be found in Fu et al. (2012). In each panel, the solid coloured curves show the mean gas-phase metallicity as a function of stellar mass at redshifts $z \sim 0,1,2,3$. The dashed curves are the fits of the observational data from AMAZE survey.

\section{References}

Fu, J., Kauffmann, G., Li, C., \& Guo, Q. 2012, MNRAS, 424, 2701 